\title{
The Fly's Eye Energetic Particle Spectrometer (FEEPS) Sensors for the Magnetospheric Multiscale (MMS) Mission
}

\author{
J.B. Blake ${ }^{1}$ - B.H. Mauk ${ }^{2}$ D.N. Baker ${ }^{3}$ - P. Carranza ${ }^{1}$ - J.H. Clemmons ${ }^{1}$ - J. Craft C $^{3}$

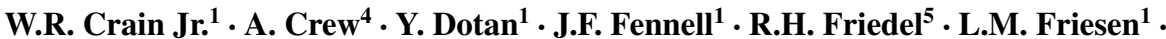 \\ F. Fuentes ${ }^{1}$ - R. Galvan ${ }^{1}$ - C. Ibscher ${ }^{1}$ - A. Jaynes ${ }^{3}$ - N. Katz ${ }^{1}$ - M. Lalic ${ }^{1}$ - A.Y. Lin ${ }^{1}$. \\ D.M. Mabry ${ }^{1}$ T. Nguyen ${ }^{1}$. C. Pancratz ${ }^{3}$ - M. Redding ${ }^{1}$ - G.D. Reeves ${ }^{5}$ - S. Smith ${ }^{4}$. \\ H.E. Spence ${ }^{4} \cdot$ J. Westlake ${ }^{2}$
}

Received: 13 December 2014 / Accepted: 13 May 2015 / Published online: 13 June 2015

(C) The Author(s) 2015. This article is published with open access at Springerlink.com

\begin{abstract}
The Energetic Particle Detector (EPD) Investigation is one of five particles and fields investigations on the Magnetospheric Multiscale (MMS) mission. This mission consists of four satellites operating in close proximity in elliptical, low-inclination orbits, and is focused upon the fundamental physics of magnetic reconnection. The Energetic Particle Detector (EPD) investigation aboard the four MMS spacecraft consists of two instrument designs, the EIS (Energetic Ion Spectrometer) and the FEEPS (Fly's Eye Electron Proton Spectrometer). This present paper describes FEEPS from an instrument physics and engineering point of view, and provides some test and calibration data to facilitate effective analysis and use of the flight data for scientific purposes.

A FEEPS consists of six Heads, each composed of two Eyes. Each eye is a particle telescope with a single silicon detector; there are nine electron eyes and three ion eyes per FEEPS. The energy coverage is from $25 \mathrm{keV}$ to $650 \mathrm{keV}$ for electrons and $45 \mathrm{keV}$ to $650 \mathrm{keV}$ for ions. Each eye has sixteen energy channels, the spacing of which can be modified by command. The fields of view and pointing of each eye are designed to provide a broad, instantaneous field of view for the twelve eyes per FEEPS.

There are two FEEPS per MMS spacecraft mounted such that the pair along with the single EIS provide more than $3 \pi$-sr instantaneous solid-angle coverage and complete coverage in the equatorial region. A twenty-second spacecraft rotation period is divided into sixty-four sectors to provide detailed temporal and spatial sampling.
\end{abstract}

\section{J.B. Blake}

jbernard.blake@aero.org

1 Space Science Applications Laboratory, The Aerospace Corporation, El Segundo, CA 90245, USA

2 The Johns Hopkins University Applied Physics Laboratory, 11100 Johns Hopkins Road, Laurel, MD 20723, USA

3 Laboratory for Atmospheric and Space Physics, 3665 Discovery Drive, Boulder, CO 80303, USA

4 Center for Earth, Oceans, and Space, University of New Hampshire, Durham, NH 03824, USA

5 Los Alamos National Laboratory, PO Box 1663, Los Alamos, NM 87545, USA 
Data are acquired in three modes: Burst Mode, the primary science mode; Fast Survey Mode; and Slow Survey Mode.

Keywords Magnetospheric Multiscale mission · Energetic electrons and protons · Fly's Eye Electron Proton Spectrometer (FEEPS)

\section{Introduction}

\subsection{FEEPS as Part of the EPD (Energetic Particle Detector) Investigation}

The Fly's Eye Energetic Electron Proton Spectrometer (FEEPS) is one of two instruments that comprise the EPD investigation. The other is the EIS (Energetic Ion Spectrometer). The EPD investigation is one of five science investigations aboard NASA's Magnetospheric Multiscale (MMS) mission. MMS consists of four identically instrumented satellites that will study the microphysics of magnetic reconnection and particle acceleration.

The EPD investigation and the EIS instrument are described in a companion paper by Mauk et al. (2014), which contains an extensive discussion of the science objectives of the investigation as well as EIS instrumental details and performance, and an overview of the FEEPS investigation. The present paper will focus upon the FEEPS instrument itself from an instrument physics and engineering point of view, and provide some test and calibration data of the engineering and flight hardware. The primary purpose of this paper is to facilitate effective analysis and use of the flight data for scientific purposes.

\subsection{Fifteen Key FEEPS Measurements Parameters}

Electrons A single FEEPS instrument contains nine sensors that makes measurements of electrons from $25 \mathrm{keV}$ to $>650 \mathrm{keV}$ in sixteen energy channels. Each sensor has a geometric factor of $0.02 \mathrm{~cm}^{2} \mathrm{sr}$.

Protons A single FEEPS contains three sensors that make measurements of protons from $45 \mathrm{keV}$ to $>650 \mathrm{keV}$ in sixteen energy channels. Each sensor has a geometric factor of $0.003 \mathrm{~cm}^{2}$ sr.

The highest data rate (burst mode) consists of a measurement sixty-four times per spacecraft rotation. The target rotation period is $3.05 \mathrm{rpm}$.

The fields of view of the sensors are somewhat complicated and are described below with illustrations.

\subsection{FEEPS Heritage}

The FEEPS instruments have a solid heritage that provides confidence that the on-orbit performance is understood.

The NASA-Polar and ESA-Cluster missions contained instruments using the same fundamental principles as FEEPS, consisting of multiple single detectors that viewed space through a collimation system that was spin scanned (Blake et al. 1995; Wilken et al. 1997). These instruments worked well for over a decade. The sensors for both missions relied upon spacecraft rotation to supply scanning in azimuth. The FEEPS eyes are spin scanned, but multiple eyes oriented at both variable elevation and azimuth are also used to provide instantaneous coverage. 
Fig. 1 Two photographs of a FEEPS instrument are shown taken from different angles to illustrate the positioning of six heads (12 eyes). Two electron heads (four eyes) in the azimuthal plane bracket the single ion head (two eyes) with the extended sun shields. The head directed up in the figures contains one electron and one ion eye. The ion eye is tilted away from normal to the plane of the box to avoid viewing the axial boom
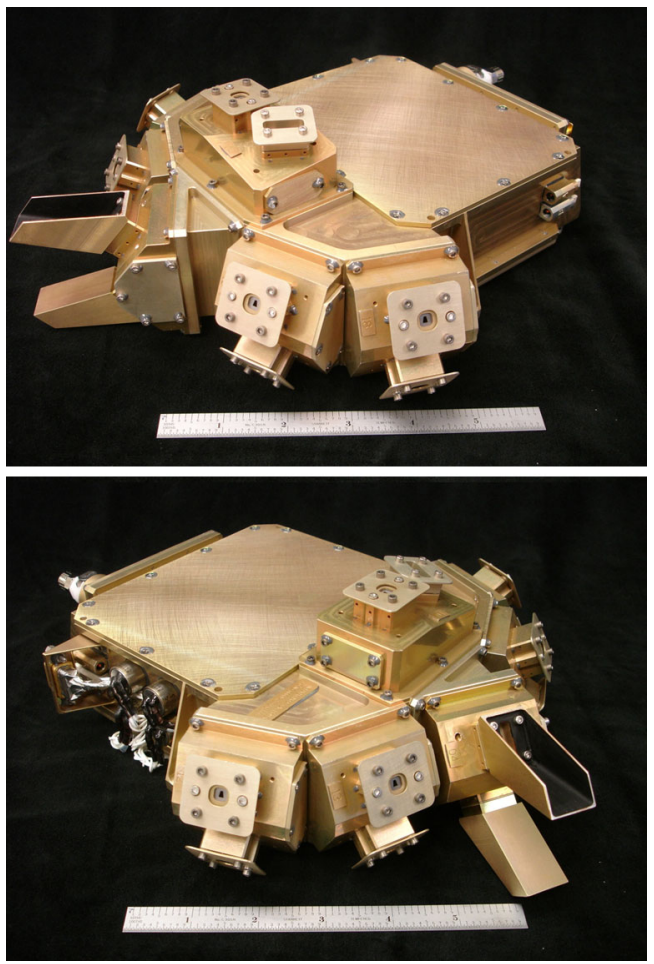

Much of the electronics design had its origin in hardware contained in the Magnetic Electron Ion Spectrometers (MagEIS) instruments (Blake et al. 2013) flown as part of the Energetic Particle, Composition, and Thermal Plasma (ECT) Suite (Spence et al. 2013) aboard the two NASA Van Allen Probes.

\subsection{FEEPS Configuration Fundamentals}

A FEEPS instrument consists of a single package that has six "heads," each of which contains two "eyes" that, in turn, contain a single, collimated silicon detector, cf. photographs of the mechanical hardware in Fig. 1. The heads in the azimuthal plane contain pairs of either electron or ion eyes whereas the head pointed either up or down contains one electron and one ion eye. There are no magnetic or electric fields, and no active collimation or veto detectors in any of the eyes. The primary purpose of FEEPS is to measure energetic electrons, with ion measurements in support of EIS. The eye labeling convention is shown in Fig. 2.

Two FEEPS instruments are aboard each of the four MMS spacecraft. The plethora of FEEPS eyes aboard each spacecraft is to provide an instantaneous measurement over more than a $3 \pi$-sr solid angle. The total number of FEEPS eyes aboard the four MMS spacecraft is ninety-six.

The two FEEPS instruments per spacecraft are mounted $180^{\circ}$ apart (Figs. 3, 4). Because of the configuration of the FEEPS fields of views (Fig. 5), the FEEPS ion detectors form effective fans (Fig. 5, left), that view in azimuth $120^{\circ}$ from each other and from the EIS ion fan (Fig. 4). The FEEPS instrument on the top of a MMS spacecraft has the top head pointing in the direction of the North ecliptic pole whereas the instrument on the bottom of 


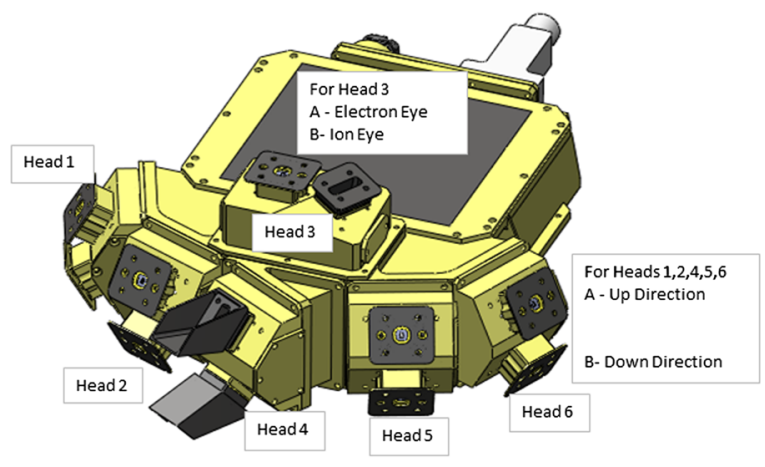

\begin{tabular}{|l|c|c|c|}
\hline \multicolumn{1}{|c|}{ Head } & Look Direction & Type & TM ID \# \\
\hline Head 1 & & & \\
\hline DAPPER E & & & \\
\hline A & Up & Electron & 1 \\
\hline B & Down & Electron & 2 \\
\hline Head 2 & & & \\
\hline DAPPER E & & & \\
\hline A & Up & Electron & 3 \\
\hline B & Down & Electron & 4 \\
\hline Head 3 & & & \\
\hline DAPPER El & & & \\
\hline A & Top & Electron & 5 \\
\hline B & Top & Ion & 6 \\
\hline Head 4 & & & \\
\hline DAPPER I & & & \\
\hline A & Up & Ion & 7 \\
\hline B & Down & Ion & 8 \\
\hline Head 5 & & & \\
\hline DAPPER E & & & \\
\hline A & Up & Electron & 9 \\
\hline B & Down & Electron & 10 \\
\hline Head 6 & & & \\
\hline DAPPER E & & & \\
\hline A & Up & Electron & 11 \\
\hline B & Down & Electron & 12 \\
\hline
\end{tabular}

Fig. 2 The FEEPS head/eye labeling convention is shown. The table gives look directions, head type and TM ID

Fig. 3 The mounting positions of the two FEEPS instruments on an MMS spacecraft are shown. The - $\mathrm{T}$ and -B simply mean Bottom and Top. The location of the EIS instrument also is shown



the spacecraft is mounted "upside down" so as to have the top head pointing in the direction of the South ecliptic pole, cf. Fig. 4.

The simple physics design of FEEPS was selected with a focus upon maximizing solidangle coverage in a short time period while keeping costs and spacecraft resources low. Such signal contamination that may occur will be addressed using the much more detailed ion observations by the EIS instrument that is part of the EPD complement. The radiation environment in the prime MMS science regions is very modest compared with that within the radiation belts; based upon earlier missions experience with similar hardware, radiation background will not be a significant issue in the analysis of data acquired in the primary science regions. 
Fig. 4 The mounting positions of the FEEPS and EIS instruments are shown as a cutaway in the $x-y$ plane to show the azimuthal fields-of-view explicitly
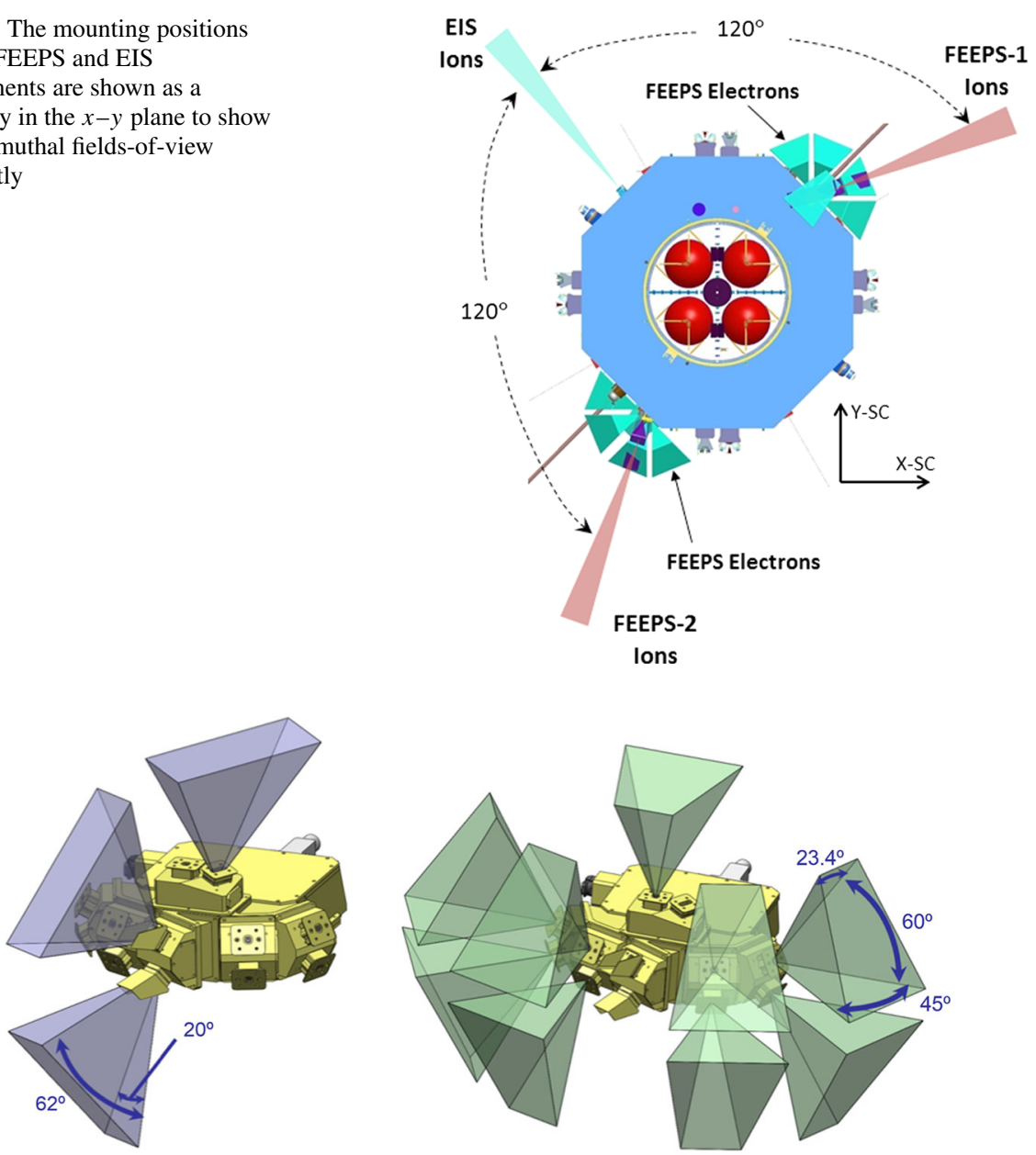

Fig. 5 The field-of-view of the three ion eyes are shown on the left. The two more equatorial eyes are cocked to avoid scanning the ecliptic plane to keep the Sun out of the field of view. The field-of-view of the nine electron eyes are shown on the right. The trapezoidal shape resulted from the efforts to minimize the blockage resulting from spacecraft appendages with little loss of solid-angle coverage. In the left hand panel it can be seen that there is $180^{\circ}$ angular coverage

\section{Principles of Operation}

Each eye is a single silicon detector telescope collimated with Mallory 1000 baffles to give the desired field of view. There are nine electron eyes and three ion eyes in each FEEPS instrument. Electron and ion eyes use different silicon detectors and different passive collimation. The electron detectors are $1 \mathrm{~mm}$ thick and therefore can stop electrons with energies of more than $650 \mathrm{keV}$ and provide some detection efficiency up to $\sim 1 \mathrm{MeV}$ due to electrons scattering as they propagate through the detector. The entrance of the electron telescope is covered with aluminum foil sufficient to stop protons with energies below $\sim 170 \mathrm{keV}$ and to block sunlight. (The detectors can see X-rays from the flaring Sun, which will be edited out during data processing. Solar X-ray background is easily detected for what it is since it would appear regularly at the period of the spacecraft rotation; the data at those instances 
Fig. 6 Cross-sections of an electron eye, left, and proton eye, right, are shown. The detectors and the final collimators are shown below
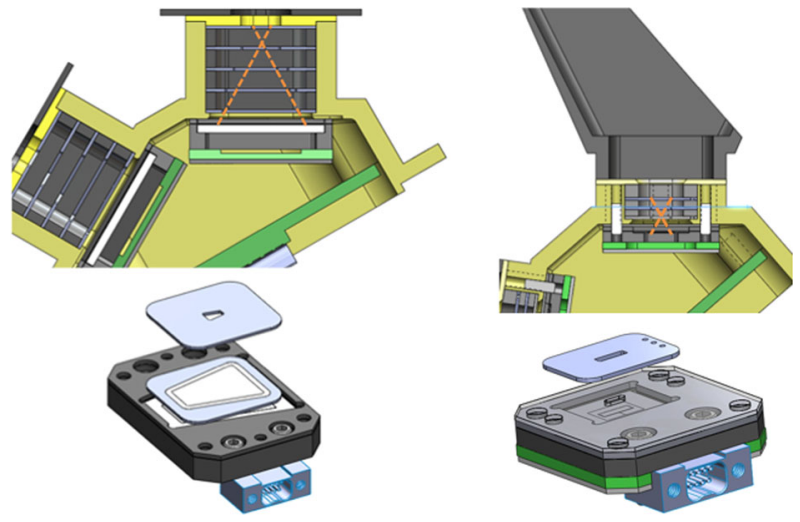

will be deleted.) The aluminum window plus system noise gives an electron energy threshold below $25 \mathrm{keV}$; the system is linear to a maximum energy of $\sim 650 \mathrm{keV}$. Energy deposits above $650 \mathrm{keV}$ go into an integral overflow channel. There are sixteen energy channels determined by pulse-height analysis of the energy deposit of each incident electron. These channel boundaries can be changed in flight if initial on-orbit observations indicate that it would be scientifically desirable to do so.

The ion detectors are nominally $9 \mu \mathrm{m}$ thick and have no covering material; they therefore are very light sensitive. The ion sensors are positioned with light baffles such that they do not scan the Sun in normal operations. The energy threshold of an ion sensor is $\sim 45 \mathrm{keV}$. This value is dominated by the electronic noise resulting from the high capacity of such a very thin detector; the detector window thickness has a negligible effect upon energy threshold. Although a substantially thicker ion detector would reduce the noise and thus lower the energy threshold; a thicker detector would substantially increase electron sensitivity. The selection of ion detector thickness was a tradeoff between noise and electron sensitivity.

A proton with an energy of $\sim 700 \mathrm{keV}$ will penetrate the silicon ion detector, and this factor sets the maximum energy that the ion sensors can detect. Higher-energy protons will deposit less than $700 \mathrm{keV}$ in the detector and thus will appear as a proton of lower energy. This background is expected to be small and can be removed by use of data acquired by the EIS sensor. Although this FEEPS sensor is called an ion sensor, it cannot distinguish between protons and other ions. But for relative abundance reasons, we know that the sensor will largely measure protons. The sixteen energy channels are determined by pulse-height analysis of the energy deposit of each incident ion.

\section{Mechanical Design}

Each FEEPS instrument uses nine thick $(1 \mathrm{~mm})$ silicon detectors to detect electrons and three thin $(9 \mu \mathrm{m})$ silicon detectors to detect ions. Pairs of detectors are housed in subassemblies, a head, along with a DAPPER (Dual Amplifier Pulse Peak Energy Rundown) ASIC (Application Specific Integrated Circuit). The subassembly housings enable the instrument to be modularized where sets of detector pairs can be swapped out with minimal disturbance to the rest of the instrument. There are three basic head types, each containing two eyes: electron-only, ion-only, and electron-ion. Figure 5 (right) shows the fields of view (FOV) of the nine FEEPS electron eyes, and Fig. 5 (left) shows the same for the ion eyes. Crosssections and perspective views of the electron (left) and ion (right) eyes are shown in Fig. 6, 

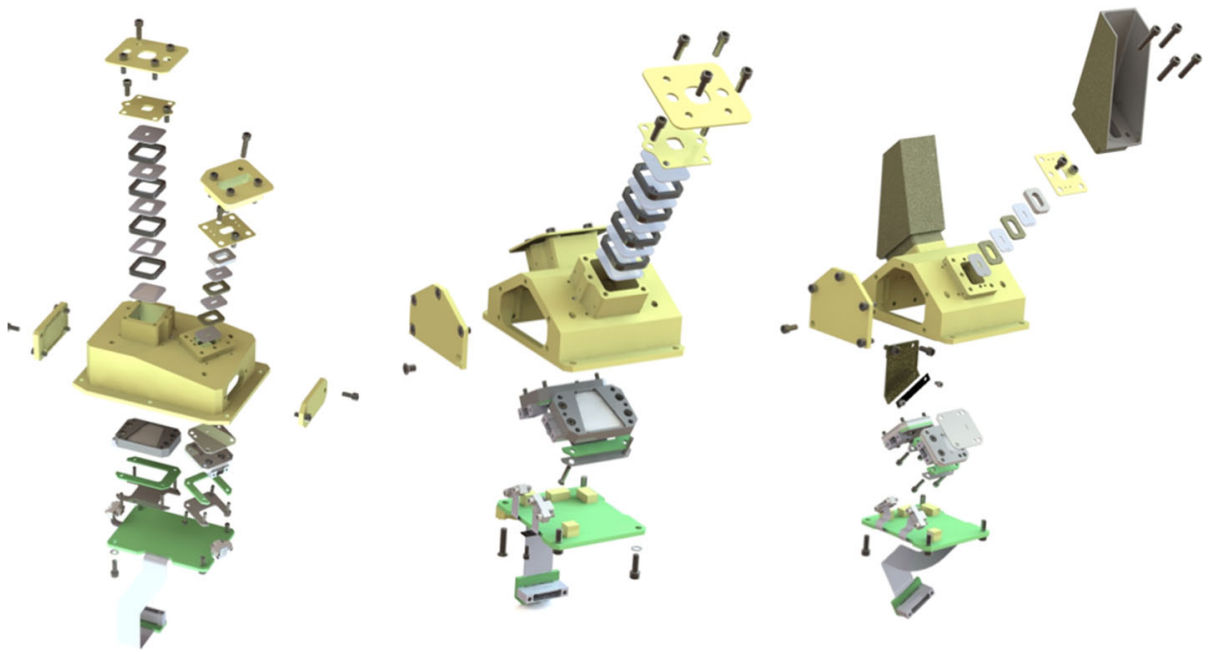

Fig. 7 Exploded views of three FEEPS heads are shown. Left to right are the electron-ion head, the electrons-only head, and the ions-only head

and exploded views of the 3 types of heads are shown in Fig. 7. The complex geometry of the FOV, especially for the electron eyes, is to minimize the solid angle of obstructions in the FOV by spacecraft appendages. Solar glint also is a concern for the ion eyes, which do not have a light-tight foil covering the detector; therefore, glint shields are used on the two equatorial ion eyes that scan near the ecliptic plane. Also, the ion eyes that view towards the north and south ecliptics have been tilted slightly away from optimum (that is away from the $\pm Z$-axes) to avoid viewing the rigid electric field booms that are aligned with the spacecraft spin axis (Fig. 5 left).

The FEEPS instrument is thermally isolated from the spacecraft decks and temperature controlled using radiators and thermostatically controlled heaters for survival. The FEEPS structure is 6061-T6 aluminum with 2.54-mm walls, a compromise between radiation shielding and weight. The weight of a single FEEPS instrument is $1.85 \mathrm{~kg}$. Additional details are provided in the Appendix.

\section{Electronic Design}

An overview of the FEEPS electronics system is seen in Fig. 8. It is composed of six detector/DAPPER heads, an Interface Board, and a Power Board. Each detector head supports two detectors. The silicon detectors will be discussed separately in a following section. A head contains a custom ASIC, called DAPPER, to amplify and read out the collected charge released in each detector. An ASIC implementation was chosen over a discrete design for its low power dissipation and small size, which allowed the chip to be located close to the detectors, thus minimizing the thermal noise component that scales with input capacitance. Figure 9 is a photograph of a head taken from the backside, showing the circuit board and harness that connects to the interface board. A plate can be seen on the near side of the head, held in place by five screws. This is an access hatch to the detector and its cable to the DAPPER. 




Fig. 8 An overview of the FEEPS electronic systems is shown

Fig. 9 A photograph of a two-electron-eye head showing the circuit board and harness

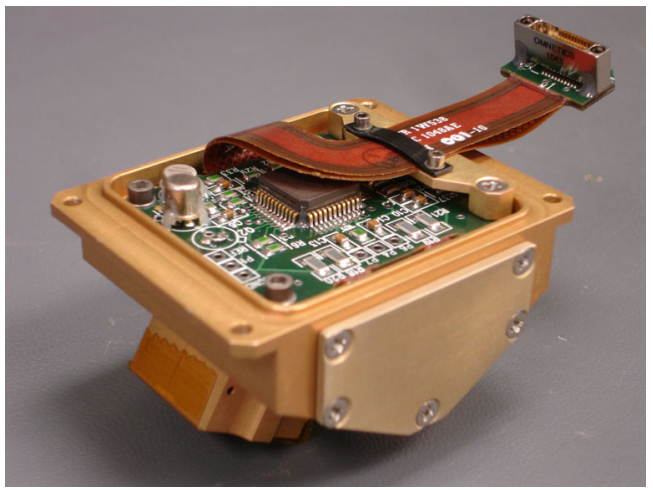

Each DAPPER amplifier string contains a charge preamplifier, pole-zero cancellation network, shaping amplifier, scaling amplifier, baseline restorer, and peak-hold amplifier. A block diagram of the DAPPER Chip is shown in Fig. 10. A Wilkinson A/D converter is formed by the interaction with the Interface Board to linearly discharge the peak-hold capacitor and measure the time it takes to return to the baseline reference voltage. In order that the output from the DAPPER not couple back to the detector inputs, the DAPPER outputs were made as current sources to reduce capacitive coupling effects. A second custom ASIC, called the SCS chip (Single-ended Current Signaling), located on the Interface Board, receives this current-mode signal and converts it back to voltage levels compatible with FPGA inputs.

The Interface Board is designed around an Actel RTSX72SU field-programmable gate array. It is a hard-wired configuration with no software element; a photograph is shown in Fig. 11. The main functions of the Interface Board are to digitize the DAPPER outputs, 


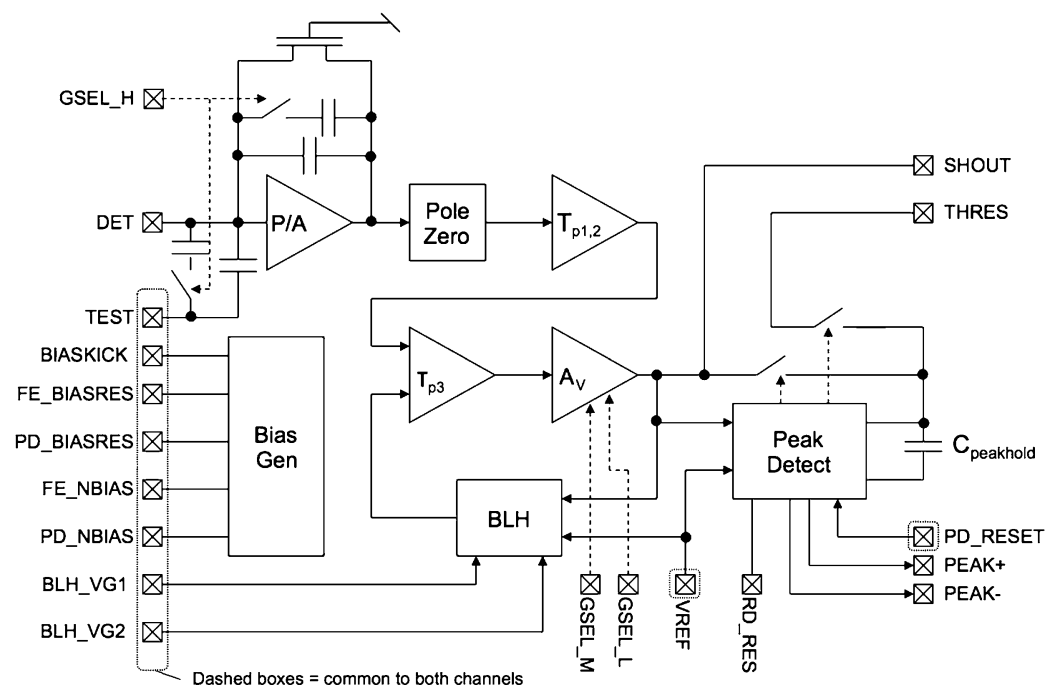

Fig. 10 A block diagram of the DAPPER ASIC

Fig. 11 A photograph of the interface board that services all twelve eyes in a single FEEPS



validate and receive command inputs from the CIDP, and generate telemetry packets at a rate of $\sim 3$ packets/second. As stated earlier, the Interface Board FPGA completes the A/D conversion by simply counting the number of system clocks in the interval of time that the peak-hold capacitor in the DAPPER is being discharged. In this way, the deadtime for each event is proportional to the size of the event. However, the overall dead-time is minimized because each DAPPER channel has its own dedicated A/D converter. Each DAPPER detector threshold can be individually programmed by the Interface Board. The Interface Board also provides a calibration pulser, housekeeping monitors, and an LVDS interface to the CIDP.

The Power Board interfaces to the spacecraft 28-V bus and utilizes an Interpoint DC/DC converter to produce the instrument low voltages. A Cockcroft-Walton multiplier operates off the $\mathrm{DC} / \mathrm{DC}$ secondary to step up the voltage and produce the $85-\mathrm{V}$ bias for the electron detectors. The bias voltage is shared among all electron detectors. The bias can be disabled, which results in about $9 \mathrm{~V}$ on each detector to maintain a small amount of depletion for 
Fig. 12 The technique employed to determine the depletion voltage of the electron detectors is shown. The ohmic surface of the detector was exposed to Am-241 alpha particles in vacuum and pulse-height spectra taken as a function of the bias voltage

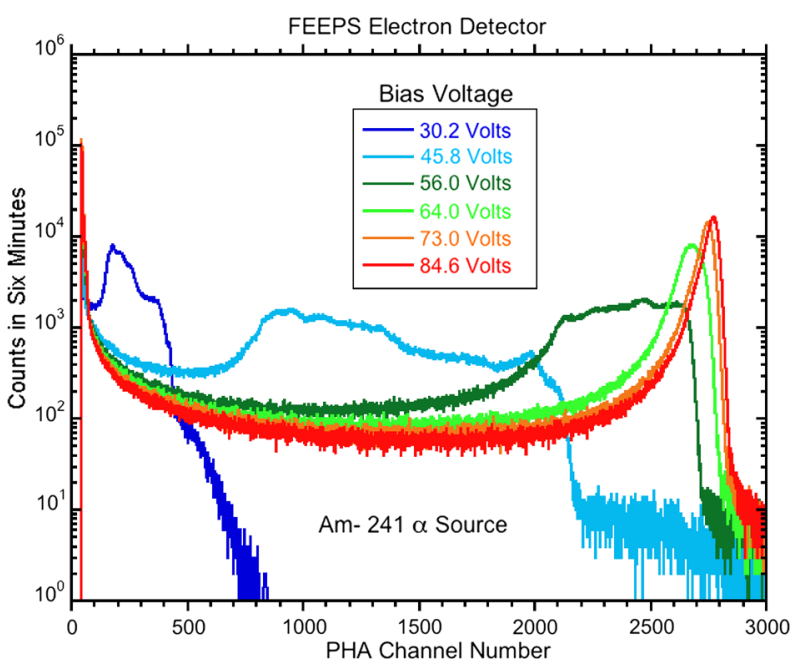

ground test. The ion detectors are thin enough to be biased with $5 \mathrm{~V}$ and therefore do not need a specific power supply but use the $5-\mathrm{V}$ bus.

\section{Silicon Detectors}

\subsection{Electron Detectors}

The FEEPS silicon electron and ion detectors were supplied by Micron Semiconductor Limited, UK.

The electron detectors have an active area that is trapezoidal in shape. The unusual shape was a result of tailoring the FOV of each eye, cf. Fig. 5; note the trapezoidal shape of the FOV. The base of the trapezoid is $10 \mathrm{~mm}$, the height is $12 \mathrm{~mm}$, and the upper side is $4.98 \mathrm{~mm}$. The detector thickness is $1000 \pm 50 \mu \mathrm{m}$. The variability of thickness is not a science issue since the detector will easily stop electrons in the energy range of primary science interest, from $25 \mathrm{keV}$ to several hundred $\mathrm{keV}$.

The depletion voltage of the electron detectors was checked by irradiating the detector in vacuum on the ohmic side with Am-241 alpha particles. All detectors were run at several bias voltages to determine the minimum voltage for total depletion. Figure 12 shows typical results.

Figure 13 shows the measured depletion voltage for approximately half of the flight electron detectors. All of the 72 detectors used in the flight hardware depleted at $75 \mathrm{~V}$ or less. As a result of these tests, the flight bias voltage for the FEEPS electron detectors was chosen to be $85 \mathrm{~V}$. Leakage currents were also checked to ascertain detector stability. Figure 13 also shows the leakage current at $170 \mathrm{~V}$, twice the flight bias voltage.

The energy resolution/noise performance of the electron detectors was checked by irradiating each flight unit with conversion electron sources, in particular, Ba-133 and Bi-207. Figure 14 is a plot of the data from one of these tests, showing energy resolution more than sufficient to meet the FEEPS needs. 
Fig. 13 The measured depletion voltage and leakage current at twice the flight bias voltage of $85 \mathrm{~V}$ is shown for approximately half of the electron detectors.

The remaining detectors behaved similarly. Detector 30, because of its high leakage current, was discarded. (Only one detector in four is labeled for clarity)
Fig. 14 The pulse-height spectrum of electrons emitted by $\mathrm{Ba}-133$ radioactive source is plotted. The detector used was one of the FEEPS flight electron detectors
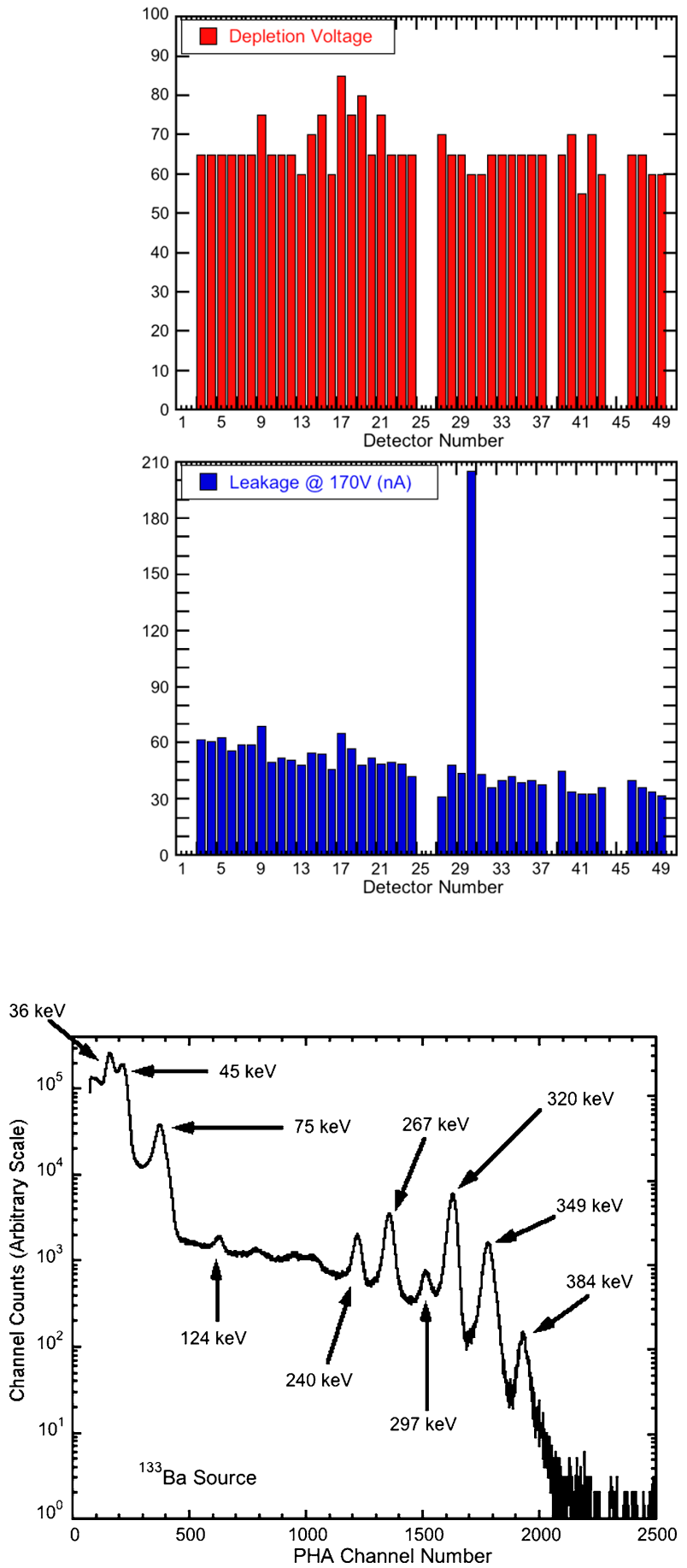
Fig. 15 The thickness of twenty-four of the ion detectors is shown as measured by alpha particle transmission. Two alpha energies were used: $3.18 \mathrm{MeV}$ and $5.8 \mathrm{MeV}$, and the mean taken

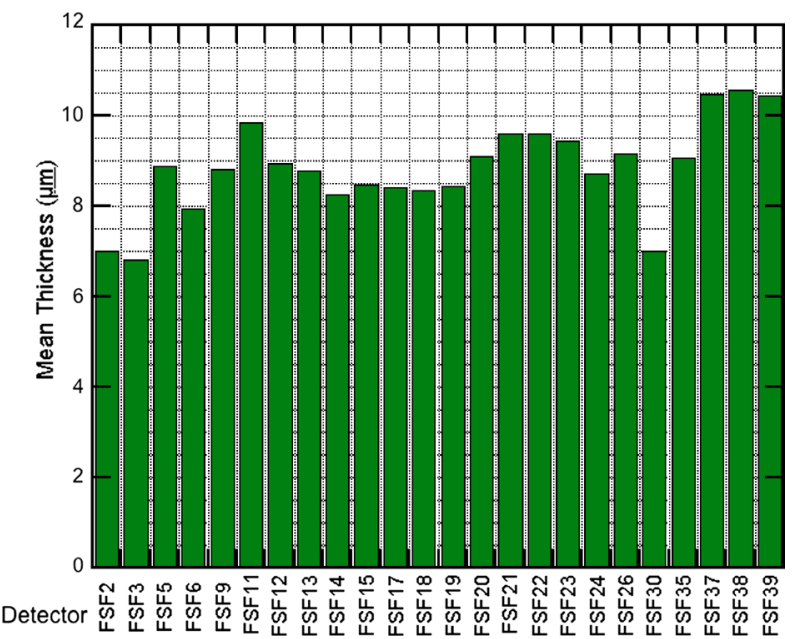

\subsection{Ion Detectors}

The ion detectors have an active rectangular area of $1.1 \mathrm{~mm} \times 3.0 \mathrm{~mm}$ and are nominally $9 \mu \mathrm{m}$ thick. The detector thickness and uniformity of thickness was measured for every flight ion detector by measuring the energy deposited from an alpha source containing Gd-148 (3.18 MeV) and Cm-244 (5.8 MeV 70\%, 5.7 MeV $30 \%)$ and using range-energy relationships to derive the silicon thickness. The estimates from the two alpha particle energies were within $\sim 10 \%$; the mean was used. Figure 15 shows the results of these measurements for 24 of the delivered flight detectors. Removing the three thinnest flight detectors from this group gave an average thickness of $9 \pm 1 \mu \mathrm{m}$. Thirty-two flight detectors were purchased from Micron, and thus thickness outliers did not need to be used.

\section{FEEPS Operational Modes}

\subsection{Telemetry Overview}

FEEPS employs a single data output format as a simplifying and cost-constraining measure. This one output goes directly to the CIDP where compression and filtering are performed, and this results in derived packet formats. There are three basic data rates and five output packet formats created in the CDIP: Burst Data, Fast Survey Data, Slow Survey Data, Housekeeping, and Low-Rate Housekeeping. Housekeeping data is output once every 10 seconds and includes analog housekeeping data, command and error counters, and status bits. Low-Rate Housekeeping is output once every 18 minutes and includes data dumps of FEEPS lookup tables.

\subsubsection{Burst Mode}

The uncompressed data allocation is $12000 \mathrm{bits} / \mathrm{s}$; the log-compressed bit rate is $11200 \mathrm{bits} / \mathrm{s}$. Like EIS, but unlike many of the sensors on MMS, the FEEPS data is acquired in a spinbased fashion, with 64 samples acquired every spin irrespective of the spin rate. For a nominal spin period of 20 seconds, 64 sectors gives a time per sector of 0.3125 seconds. Each 
of the 12 eyes has 16 energy channels derived from the 256-channel pulse-analysis within the instrument. Data for each eye/channel is compressed from raw 16-bit resolution by the CIDP to form a 12-bit result. In addition, each eye has a livetime channel, also 12 bits. There are three fast sampling channels called Integral Rates, which provide $\sim 10 \mathrm{~ms}$ sampling for any combination of eyes/channels as defined in flight-programmable lookup tables.

\subsubsection{Fast Survey Mode}

The data allocation is $666 \mathrm{bits} / \mathrm{s}$, with a log-compressed rate of 618 bits/s. Only 5 of the 8 electron eyes are used; all of the ion eyes are used. Thus, 8 of the 12 eyes remain in operation. These 8 eyes each have a livetime channel of 12 bits. The sample rate is reduced 8 -fold, thus 8 sectors per spin rather than the 64 sectors per spin of the burst mode.

\subsubsection{Slow Survey Mode}

The slow survey mode has a bit allocation of $10 \%$ of the fast survey mode. It is obtained simply by using only one spin in ten.

\section{FEEPS Calibrations and Performance}

It was not practical to exhaustively calibrate all ninety-six FEEPS eyes with energetic particles over the entire energy ranges. Sensor variability was determined by laboratory testing during the build cycle by the use of pulsers and gamma sources during thermal and thermal vacuum testing. Eyes that differed by more than several percent from the average were not used in the flight hardware. The eyes used in the detailed accelerator testing described below were typical representatives of the flight hardware.

\subsection{Electron Eyes}

\subsubsection{Electron Measurements}

A pair of electron eyes was exposed to monoenergetic electrons at the Goddard Space Flight Center, the Johns Hopkins Applied Physics Laboratory, and The Aerospace Corporation. Calibration facilities at multiple institutions were used in order to get the necessary energy and species coverage needed. The electron beams varied in energy from $15 \mathrm{keV}$ (below threshold) to above $1 \mathrm{MeV}$ (above sensor saturation).

\subsubsection{Conversion Electron and $\beta$-Spectra Response}

A straightforward test of the performance of an electron eye is to expose it to a beta source. The two sources used in general were Ni-63 and C-14. These two isotopes were selected because they decay to the ground state of the daughter isotope and thus emit no gammas, and the beta energy spectra are soft and nicely cover the electron energy range of prime scientific interest for MMS. The endpoint energy of Ni-63 is $67 \mathrm{keV}$ and of C-14 is $156 \mathrm{keV}$.

The results of a representative test are shown in Fig. 16; the electron eye was exposed to the two sources in vacuum. The beta spectra are cleanly seen in both cases including the endpoint energy. The noise threshold can be seen to be $\sim 16 \mathrm{keV}$.

The beta spectra shown in Fig. 16 were taken without the aluminum light-tight foil being in place. The effect of this foil was examined by exposing an electron eye to a Ni-63 source with and without an aluminum foil. The results are shown in Fig. 17. 
Fig. 16 Pulse height spectra taken using Ni-63 and C-14 sources are shown for a representative electron eye



Fig. 17 Pulse-height spectra taken using a Ni-63 source in a representative electron eye are shown. The spectra were taken with and without the Al foil used in the flight hardware to block sunlight and stop protons with energies below $\sim 170 \mathrm{keV}$. It can be seen that the foil has very little effect for electrons above $\sim 25 \mathrm{keV}$, the nominal FEEPS electron threshold

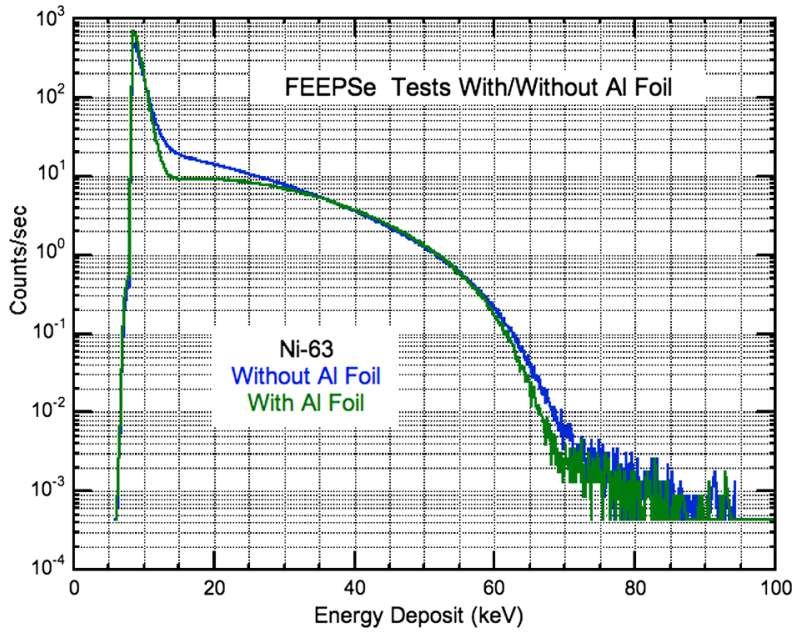

\subsubsection{Electron Accelerator Calibrations}

Electron accelerators were used to provide calibration data over the entire operational energy range. Figure 18 shows calibration data in the upper portion of an electron eye passband, above $100 \mathrm{keV}$. Excellent energy resolution is seen, and the energy peaks have little out-ofband response.

Figure 19 shows electron calibration data taken near the energy threshold. It can be seen that the energy peaks are well resolved from the noise at an incident electron energy of $\sim 30 \mathrm{keV}$. As the electron energy decreases, the low-energy side of the pulse-height distribution moves into the system noise. Thus, by $25 \mathrm{keV}$, a significant fraction of the pulse height distribution is in the noise region, and the effective efficiency of that channel drops below unity.

It should be noted that these calibrations were made at laboratory room temperature. The on-orbit temperature is expected to be significantly less, and therefore the system noise will drop as was shown during testing in thermal and thermal vacuum testing. During com- 
Fig. 18 Normalized calibration data are plotted for an electron eye irradiated by electrons with six discrete energies above $100 \mathrm{keV}$. The location of the overflow channel at $650 \mathrm{keV}$ is indicated. The FWHM of the energy peaks was $\sim 14 \mathrm{keV}$

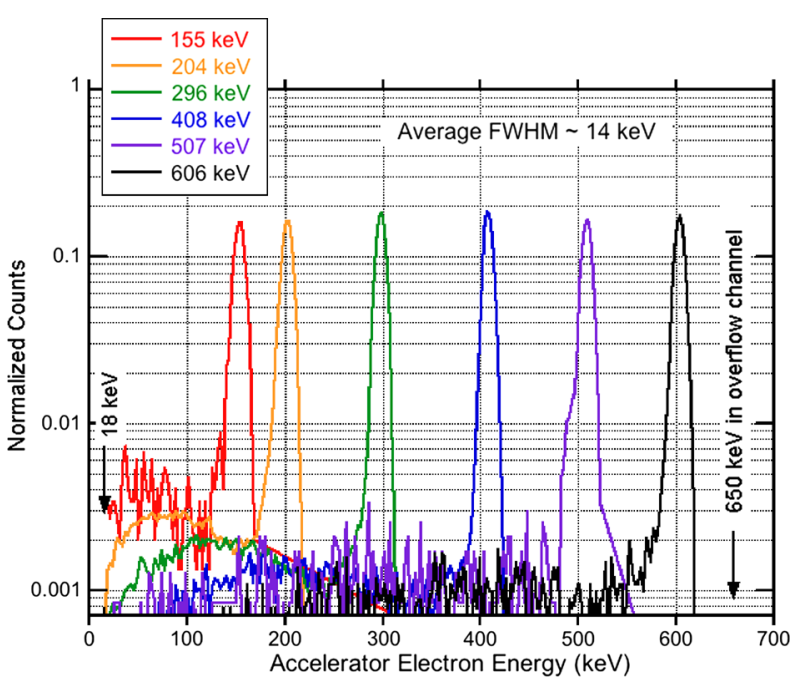

Fig. 19 Normalized calibration data take with a representative electron eye are shown for electrons with energies near the noise threshold of $\sim 20 \mathrm{keV}$.

These data were taken at room temperature. It is expected that the noise threshold by a few $\mathrm{keV}$ at in-flight temperatures

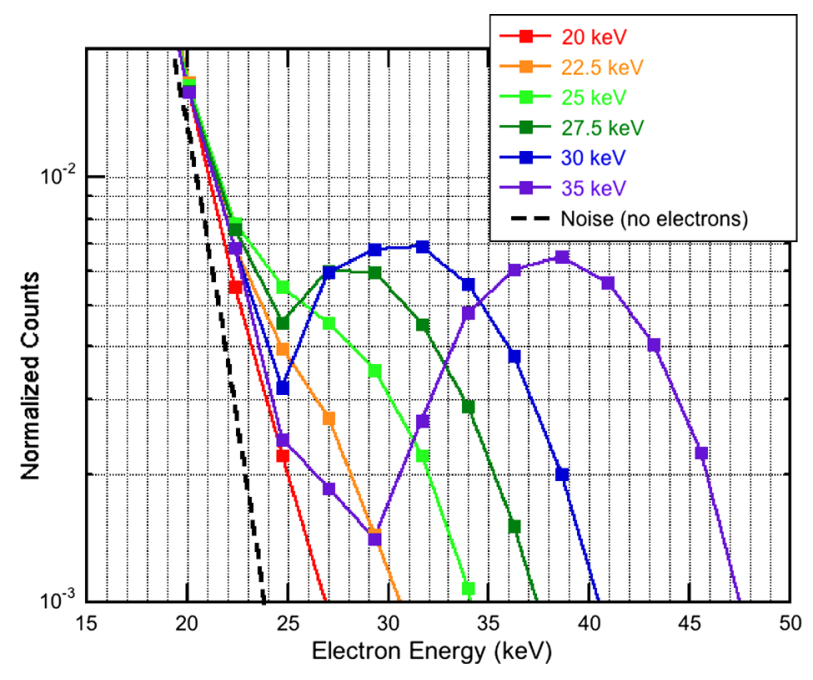

missioning, both radiation belt electrons and the on-board pulser will be used to determine efficiencies near the energy threshold.

\subsubsection{Proton Accelerator Calibrations of Electron Eyes}

Protons (ions) could be a significant source of background in the FEEPS electron eyes depending upon their abundance and energy spectra relative to the electron population. It is important to understand the response of the electron eyes to protons to determine under what circumstances such background might be important. To that end, an electron eye was exposed to a proton beam with energies from that just sufficient to penetrate the aluminum foil that serves as a light shield to several hundred keV (see Fig. 20). The light shield degrades the incident proton beam, resulting in an energy deposit in the detector less than that of the incident proton beam. 
Fig. 20 The response of an electron eye to protons is shown for four incident proton energies from the aperture foil penetration threshold of $\sim 170 \mathrm{keV}$ to $408 \mathrm{keV}$

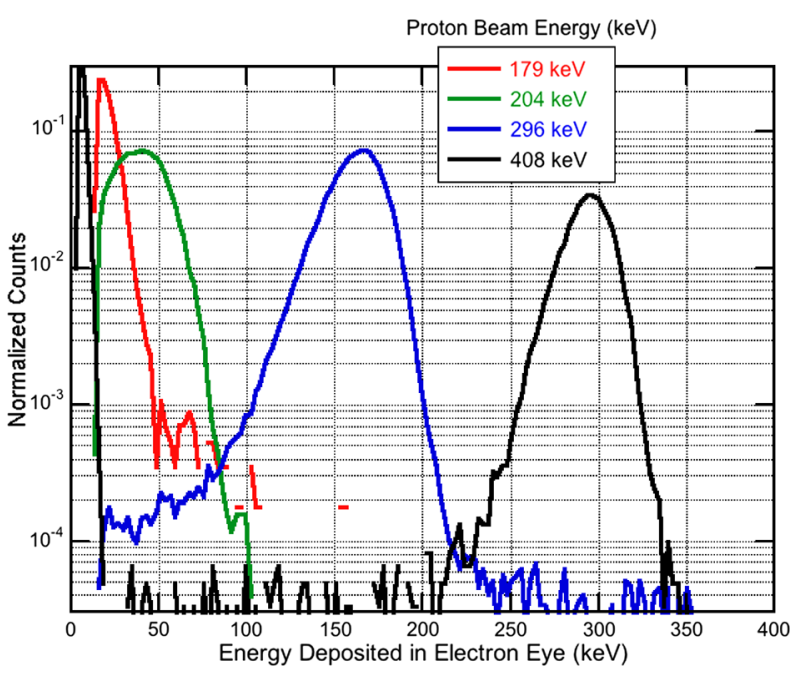

The ion data from the FEEPS ion eyes and from the EIS instrument will be used to remove this background where necessary using the proton energy spectra measured by EIS to determine the intensity of protons causing this background. It is expected that in the primary MMS science regions, this correction will be small to negligible due to the expected steep proton energy spectra.

\subsubsection{Response to Energetic Solar Particles (ESP)}

Energetic solar particles will have access to the MMS satellites when the vehicles are in the mission science regions. ESP particles range in energies from those within the passbands of the FEEPS instrument to those with sufficient energy to easily penetrate the FEEPS instrument structure and even through the MMS spacecraft. The ESP particles within the passbands can be distinguished by the temporal history and from other data sources such as ACE and GOES satellites.

Electron and ion heads were exposed to protons in the 10 to $50 \mathrm{MeV}$ energy range using the Lawrence Berkeley National Laboratory (LBNL) 88-in. Cyclotron. Except for protons entering through entrance collimator, the response was nil.

\subsubsection{Response to the Galactic Cosmic Rays (GCR)}

During the prime science portion of the MMS orbit, the spacecraft will be exposed to the unimpeded GCR flux. The GCR will cause a uniform background count rate in the electron eyes of about $2 / \mathrm{sec}$. (Uniform in that the changes in the GCR intensity are on a much longer timescale than MMS science variability.) A Geant 4 calculation was carried out in order to estimate the magnitude of this background. (Geant 4 is a Monte Carlo radiation transfer code.) Figure 21 presents an estimate of the geometric factor of a FEEPS electron detector to the GCR. In the energy deposit range of FEEPS, science interest is below $\sim 700 \mathrm{keV}$; the geometric factor changes little. Commissioning observations of the GCR background will be used to correct the FEEPS electron data in the database, in particular to ensure that it does not appear to the casual observer that there is a steady-state population of energetic electrons surrounding the MMS spacecraft. 
Fig. 21 The result of a Geant 4 calculation of the response of a FEEPS electron detector to the GCR is shown. The geometric factor is seen to be almost constant over the energy-deposit range of a FEEPS electron eye

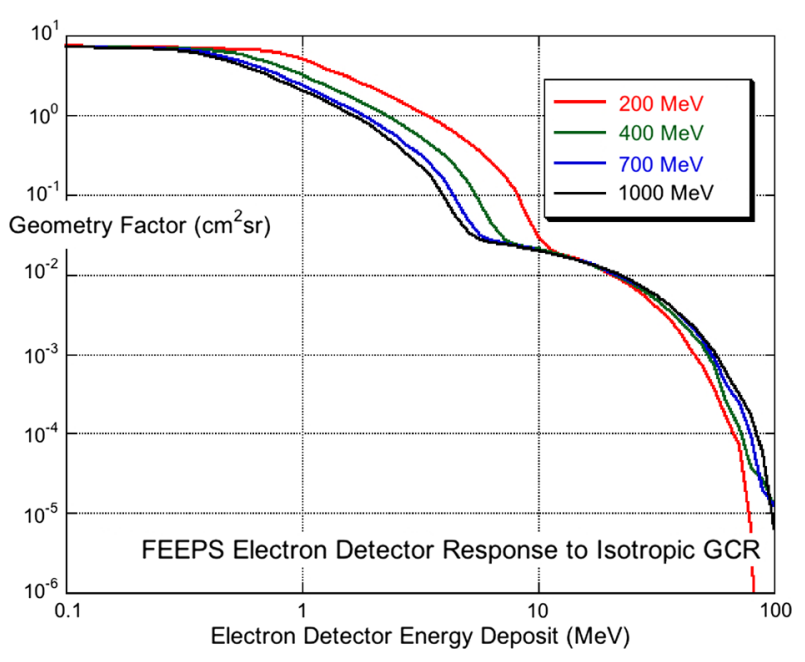

Fig. 22 The results of a Geant 4 calculation of the response of an ion eye to electrons are shown. The shaded area is the energy region of interest for FEEPS



The ion eyes are sufficiently thin that the GCR do not deposit enough energy to create a significant background.

\subsection{Ion Eyes}

\subsubsection{Geant4 Studies of Electron Sensitivity}

The issue of the response of a ion eye to incident electrons was studied both computationally with Geant 4 simulations and experimentally using electron sources and accelerator beams.

Figure 22 is an example of the results of Geant 4 simulations of a FEEPS eye to electrons. At a FEEPS ion eye energy threshold of $50 \mathrm{keV}$, a $50-\mathrm{keV}$ electron has an efficiency of $\sim 30 \%$, but by the time the incident electron energy is $70 \mathrm{keV}$, the efficiency has dropped to $\sim 4 \%$, and by $90 \mathrm{keV}$, the efficiency is well under $2 \%$. 
Fig. 23 The response of a FEEPS ion eye to protons over most of the energy range of ion eyes is shown. The data were taken with the GSFC van de Graaf

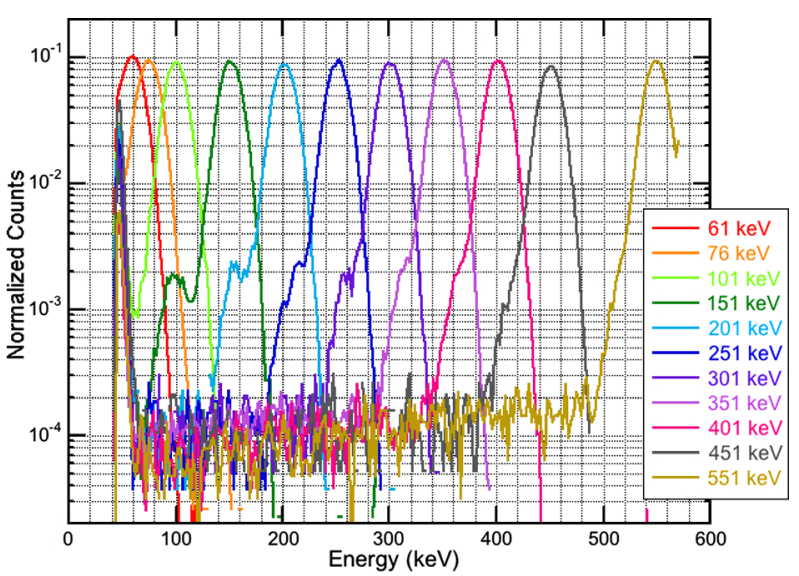

Fig. 24 Pulse-height spectra are shown for protons around $1 \mathrm{MeV}$ that penetrate the ion detector and thus do not deposit their entire energy. Two spectra of stopping protons within an ion eye passband are shown for comparison

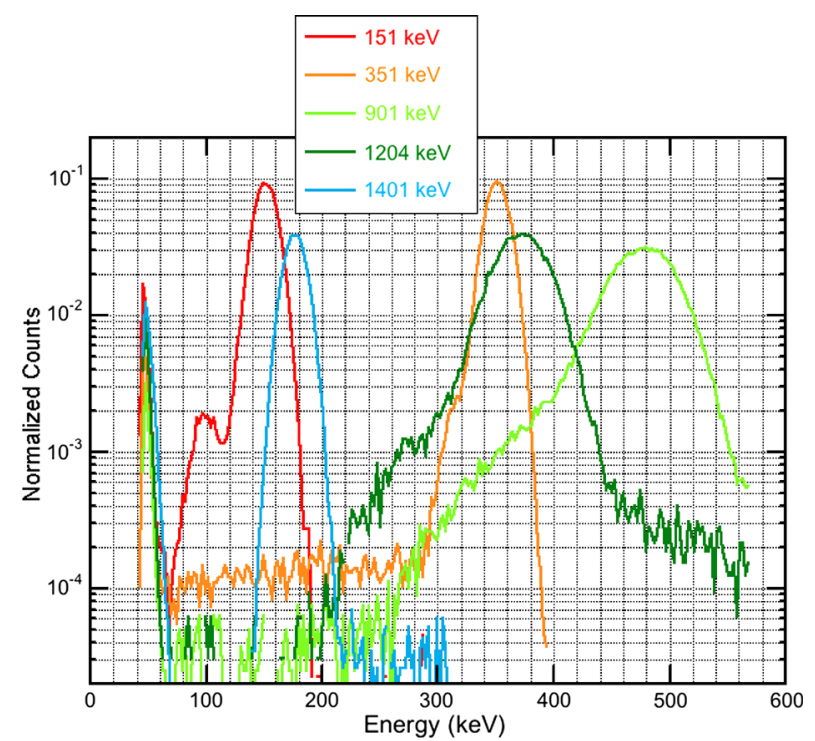

\subsubsection{Accelerator Ion Testing}

Representative FEEPS ion eyes were tested using the GSFC and APL ion accelerators. The eyes were exposed to protons from the FEEPS threshold of $\sim 50 \mathrm{keV}$ to $1400 \mathrm{keV}$, the latter being about twice the energy required to penetrate the thin ion detector. These tests provided detailed knowledge of the response of ion eyes to low-energy ions. Figure 23 shows an example of the observations and shows pulse-height spectra from proton beam energies of $61 \mathrm{keV}$ to $551 \mathrm{keV}$.

Protons above the detector penetration energy give energy deposits below the incident energy. Figure 24 shows pulse-height spectra for stopping $(<600 \mathrm{keV})$ and penetrating $(>700 \mathrm{keV}$ ) protons. Background due to penetrating protons can be removed during data processing by using EIS proton spectra as well as the proton spectra from FEEPS itself.

Acknowledgements This work was supported by the Johns Hopkins Applied Physics Laboratory under Contract 792084N/E99017JD. 


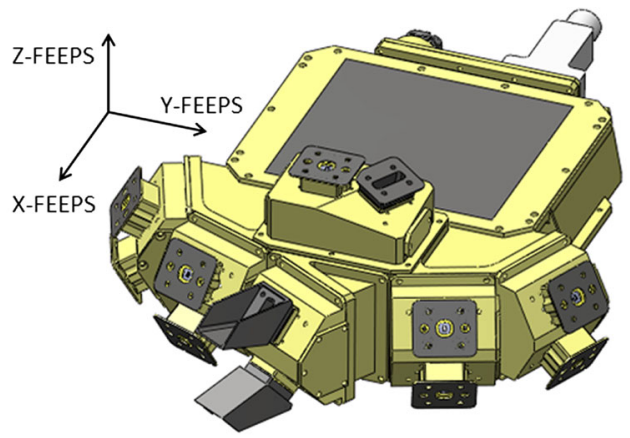

\begin{tabular}{|c|ccc|cc|c|ccc|}
\hline ID\# & Head & Orientation & Species & Azimuth & Elevation & $\begin{array}{c}\text { Weighted } \\
\text { Elevation }\end{array}$ & Unit-X & Unit-Y & Unit-Z \\
\hline 1 & 1 & Up & Electons & -67.5 & 30 & 25 & 0.347 & -0.837 & 0.423 \\
2 & 1 & Down & Electons & -67.5 & -30 & -25 & 0.347 & -0.837 & -0.423 \\
3 & 2 & Up & Electons & -22.5 & 30 & 25 & 0.837 & -0.347 & 0.423 \\
4 & 2 & Down & Electons & -22.5 & -30 & -25 & 0.837 & -0.347 & -0.423 \\
5 & 3 & Top & Electons & 0 & 90 & 95 & -0.087 & 0.000 & 0.996 \\
6 & 3 & Top & lons & 60 & 78 & 78 & 0.104 & 0.180 & 0.978 \\
7 & 4 & Up & lons & -30 & 41 & 41 & 0.654 & -0.377 & 0.656 \\
8 & 4 & Down & lons & -30 & -41 & -41 & 0.654 & -0.377 & -0.656 \\
9 & 5 & Up & Electrons & 22.5 & 30 & 25 & 0.837 & 0.347 & 0.423 \\
10 & 5 & Down & Electrons & 22.5 & -30 & -25 & 0.837 & 0.347 & -0.423 \\
11 & 6 & Up & Electrons & 67.5 & 30 & 25 & 0.347 & 0.837 & 0.423 \\
12 & 6 & Down & Electrons & 67.5 & -30 & -25 & 0.347 & 0.837 & -0.423 \\
\hline
\end{tabular}

Fig. 25 The FEEPS coordinate system is shown along with the unit vectors for each of the 12 eyes in the FEEPS coordinate system. The "elevation" angle is the angle made by unit vectors pointing normal to the front faces of the SSD's. The "Weighted Elevation" takes into account the non-symmetric shape of the electron fields of view (centered on the centroid of the solid angle viewed)

Open Access This article is distributed under the terms of the Creative Commons Attribution 4.0 International License (http://creativecommons.org/licenses/by/4.0/), which permits unrestricted use, distribution, and reproduction in any medium, provided you give appropriate credit to the original author(s) and the source, provide a link to the Creative Commons license, and indicate if changes were made.

\section{Appendix: FEEPS Miscellaneous Specifications}

\section{A.1 Each FEEPS Instrument}

Total Mass: $\quad 1.85 \mathrm{~kg}$

Operational Power: 1.73 watts

Data Rate: $\quad$ Burst: 12 kbps; Fast Survey: 0.67 kbps; Slow Survey: 0.067 kbps.

\section{A.2 FEEPS Detectors}

Electron Eye Geometric Factor: $\quad 0.02 \mathrm{~cm}^{2} \mathrm{sr}$

Electron SSD thickness: $\quad 1 \mathrm{~mm}$

Electron SSD size: $\quad$ Trapezoid base: $10 \mathrm{~mm}$, top: $4.98 \mathrm{~mm}, \mathrm{H}: 12 \mathrm{~mm}$

Electron foil: $\quad 1.3$ microns aluminum

Electron Pinhole Size:* Trapezoid base: $1.86 \mathrm{~mm}$, top: $0.58 \mathrm{~mm}, \mathrm{~h}: 2.39 \mathrm{~mm}$

Electron SSD to pinhole distance: $1.06 \mathrm{~cm}$

Ion Eye Geometric Factor: $\quad 0.003 \mathrm{~cm}^{2} \mathrm{sr}$

Ion SSD thickness: $\quad 9$ microns 
Ion SSD size:

Ion Pinhole Size:

Ion SSD to pinhole distance:
$3 \mathrm{~mm} \times 1 \mathrm{~mm}$

$3 \mathrm{~mm} \times 0.9 \mathrm{~mm}$

$0.5 \mathrm{~cm}$

* Note: to obtain the trapezoidal fields of view, the pinhole trapezoidal shape is upside-down with respect to the SSD trapezoidal shape.

\section{A.3 FEEPS Detector Configurations and Viewing}

Figure 2 shows the labeling for the 12 different fields of view on each FEEPS instrument, 9 electron views and 3 ion views.

Note that all 12 of these views are used for generating Burst data; however, only a subset is used in the generation of Survey data. For Survey data all of the ion views are used, but only the electron views with IDs 3, 4, 5, 11, and 12 are used (see the IDs in Fig. 2).

Note that the transformation of vectors from the FEEPS-T (FEEPS-payload-deck or the "up" direction) to the spacecraft coordinate system (shown in Figs. 3, 4) is achieved using: $V(s c)=T 1($ FEEP-T $) \cdot V($ FEEPS-T) using:

$$
T 1 \text { (FEEPS-T) }=\left[\begin{array}{ccc}
1 / \sqrt{2} & -1 / \sqrt{2} & 0 \\
1 / \sqrt{2} & 1 / \sqrt{2} & 0 \\
0 & 0 & 1
\end{array}\right]
$$

Similarly, the transformation of vectors from the FEEPS-B (FEEPS-bottom-deck) to the spacecraft coordinate system is achieved using: $V(s c)=T 2$ (FEEP-B) $\cdot V$ (FEEPS-B) using:

$$
T 1 \text { (FEEPS-2) }=\left[\begin{array}{ccc}
-1 / \sqrt{2} & -1 / \sqrt{2} & 0 \\
-1 / \sqrt{2} & 1 / \sqrt{2} & 0 \\
0 & 0 & -1
\end{array}\right]
$$

\section{References}

J.B. Blake, J.F. Fennell, L.M. Friesen, B.M. Johnson, W.A. Kolasinski, D.J. Mabry, J.V. Osborn, S.H. Penzin, E.R. Schnauss, H.E. Spence, D.N. Baker, R. Belian, T.A. Fritz, W. Ford, B. Laubscher, R. Stiglich, R.A. Baraze, M.F. Hilsenrath, W.L. Imhof, J.R. Kilner, J. Mobilia, D.H. Voss, A. Korth, M. Gull, K. Fisher, M. Grande, D. Hall, CEPPAD_comprehensive energetic particle and pitch angle distribution experiment on POLAR. Space Sci. Rev. 71, 531 (1995)

J.B. Blake, P.A. Carranza, S.G. Claudepierre, J.H. Clemmons, W.R. Crain Jr., Y. Dotan, J.F. Fennell, F.H. Fuentes, R.M. Galvan, J.S. George, M.G. Henderson, M. Lalic, A.Y. Lin, M.D. Looper, D.J. Mabry, J.E. Mazur, B. McCarthy, C.Q. Nguyen, T.P. O’Brien, M.A. Perez, M.T. Redding, J.L. Roeder, D.J. Salvaggio, G.A. Sorensen, H.E. Spence, S. Yi, M.P. Zakrzewski, The magnetic electron ion spectrometer (MagEIS) instruments aboard the radiation belt storm probes (RBSP) spacecraft. Space Sci. Rev. (2013). doi:10.1007/s11214-013-9991-8

B.H. Mauk, J.B. Blake, D.N. Baker, J.H. Clemmons, G.D. Reeves, H.E. Spence, S.E. Jaskulek, C.E. Schlemm, L.E. Brown, S.A. Cooper, J.V. Craft, J.F. Fennell, R.S. Gurnee, C.M. Hammock, J.R. Hayes, P.A. Hill, G.C. Ho, J.C. Hutcheson, A.D. Jacques, S. Kerem, D.G. Mitchell, K.S. Nelson, N.P. Paschalidis, E. Rossano, M.R. Stokes, J.H. Westlake, The energetic particle detector (EPD) investigation and the energetic ion spectrometer (EIS) for the magnetospheric multiscale (MMS) mission. Space Sci. Rev. (2014). doi:10.1007/s11214-014-0055-5

H.E. Spence, G.D. Reeves, D.N. Baker, J.B. Blake, M. Bolton, S. Bourdarie, A.A. Chan, S.G. Claudepierre, J.H. Clemmons, J.P. Cravens, S.R. Elkington, J.F. Fennell, R.H.W. Friedel, H.O. Funsten, J. Goldstein, J.C. Green, A. Guthrie, M.G. Henderson, R.B. Horne, M.K. Hudson, J.-M. Jahn, V.K. Jordanova, S.G. Kanekal, B.W. Klatt, B.A. Larsen, X. Li, E.A. MacDonald, I.R. Mann, J. Niehof, T.P. O’Brien, T.G. Onsager, D. Salvaggio, R.M. Skoug, S.S. Smith, L.L. Suther, M.F. Thomsen, R.M. 
Thorne, Science goals and overview of the radiation belt storm probes (RBSP) energetic particle, composition, and thermal plasma (ECT) suite on NASA's Van Allen probes mission. Space Sci. Rev. (2013). doi:10.1007/s11214-013-0007-5

B. Wilken, W.I. Axford, I. Daglis, P. Daly, W. Güttler, W.H. Ip, A. Korth, G. Kremser, S. Livi, V.M. Vasyliunas, J. Woch, D.N. Baker, R.D. Belian, J.B. Blake, J.F. Fennell, L.R. Lyons, H. Borg, T.A. Fritz, F. Gliem, R. Rathje, M. Grande, D. Hall, K. Kecsueméty, S. McKenna-Lawlor, K. Mursula, P. Tanskanen, Z. Pu, I. Sandahl, E.T. Sarris, M. Scholer, M. Schulz, F. Sørass, S. Ullaland, RAPID—the imaging energetic particle spectrometer on CLUSTER. Space Sci. Rev. 79, 399 (1997) 